\title{
DISRUPTION POTENTIAL OF THE DISTRIBUTED LEDGER TECHNOLOGY WITHIN THE ECONOMY OF LATVIA
}

\author{
Natalija Kostrikova ${ }^{1}$, MBA; Baiba Rivza ${ }^{2}$, Dr. habil. oec
}

${ }_{1,2}$ Latvia University of Life Science and Technologies

\begin{abstract}
The main research objective is to identify and analyse significant economic sectors subject to potential disruption from the distributed ledger technology (DLT) within the economy of Latvia. The tasks are 1) to identify sectors subject to disruption from DLT in line with global technological developments, business readiness trends and distributed ledger use cases, 2) to identify sectors generating the most significant output within the economy of Latvia, 3) to analyse interconnections of global trends related to DLT and the areas of potential disruptions to the identified sectors within the economy of Latvia. The research concluded that economic sectors with the most significant output within the economy of Latvia are all subject to disruption from distributed ledger use cases and global DLT trends in the short, medium or long term.
\end{abstract}

Key words: distributed ledger technology, blockchain, disruption.

JEL code: 033.

\section{Introduction}

Alongside more frequent appearance of words 'blockchain' and 'distributed ledger technology' (DLT) in research as well as deployment of DLT use cases beyond crypto-currencies and initial coin offerings (ICOs), the broader community has ultimately reached the point when blockchain's association with purely crypto-currencies and Bitcoin is fading away. By business leaders, DLT is No longer seen as a stand-alone technology, but rather as a business enabler, that can not only provide faster, more secure and cost-efficient transactions, but also streamline democratization of trust and fundamentally reshape existing business models. DLT can record transactions, track the origins of goods, store medical records, verify information, facilitate automatic payments through implementation of smart contracts and provide many other opportunities to ensure transparency, security and operational efficiency. However, it brings No benefit unless it is linked to a solid use case, thus, serving as a Trust-as-a-Service (TaaS) that improves ways of trust management within cloud environments.

The research is focused on identifying potential for emergence of DLT use cases beyond cryptocurrencies and ICOs that can bring the most significant economic impact measured by output to the economy of Latvia. The research does not specifically analyse fintech DLT solutions since those solutions are considered to be cross-sectorial, thus, requiring a separate in-depth analysis. The content analysis method is applied for identification of DLT use cases within recent research papers as well as web-sites of actual DLT applications, DLT communities and DLT consortia. The relevant content is further analysed from the use case perspective within appropriate economic sectors. The descriptive statistics method is applied for identification and visualisation of the sectors that bring significant contribution to the economy of Latvia derived from quantitative statistical data. Inductive reasoning is further applied for concluding on the disruption potential to the identified sectors within the economy of Latvia based on the time frame approximation, which is required for the identified DLT use cases and trends to disrupt the established business models globally, regionally and locally. The time frame approximation exercise is based on the assumption that identified DLT use cases have already been put into use and identified DLT trends will be put into use in line with Rogers' innovation diffusion theory that implies reaching the 4th stage of innovation decision-making process named 'Implementation' (Rogers, 1962). The reasoning process presumes that use cases that are

\footnotetext{
${ }^{1}$ kostrikova.natalia@gmail.com

2 baiba.rivza@llu.Iv
} 
currently present in Latvia are likely to disrupt relevant economic sectors in short term, use cases that are present in Central and Eastern Europe are likely to disrupt relevant economic sectors in medium term, uses cases in other geographies and overall global trends are likely to disrupt relevant economic sectors in long term, whilst non-existence of use cases and global trends would presume No disruption potential in relevant sectors.

\section{Research results and discussion}

\section{Analysis of global and regional DLT trends and use cases}

According to Deloitte's 2018 global blockchain survey of more than 1,000 blockchain-savvy executives, a majority (74 percent) of respondents report that their organizations see a 'compelling business case' for the use of blockchain technology, however, only 34 percent say their company has initiated some sort of blockchain deployment (Deloitte, 2018). This certainly reflects not only the existence of business rationale for DLT implementation (since blockchain is a form of DLT), but also a potential for narrowing the gap between 'Decision' and 'Implementation' stages according to Rogers' innovation diffusion theory (Rogers, 1962) through emergence of new and further development of existing DLT use cases in short to medium term globally. The same survey (Deloitte, 2018) indicates that there is a clear market sentiment that blockchain technology is expected to disrupt an array of industries with Automotive, Oil\&Gas and Life sciences topping the list (73\%, $72 \%$ and $72 \%$ of respondents accordingly) and Public sector being at the bottom of potential disruption (46\% of respondents).

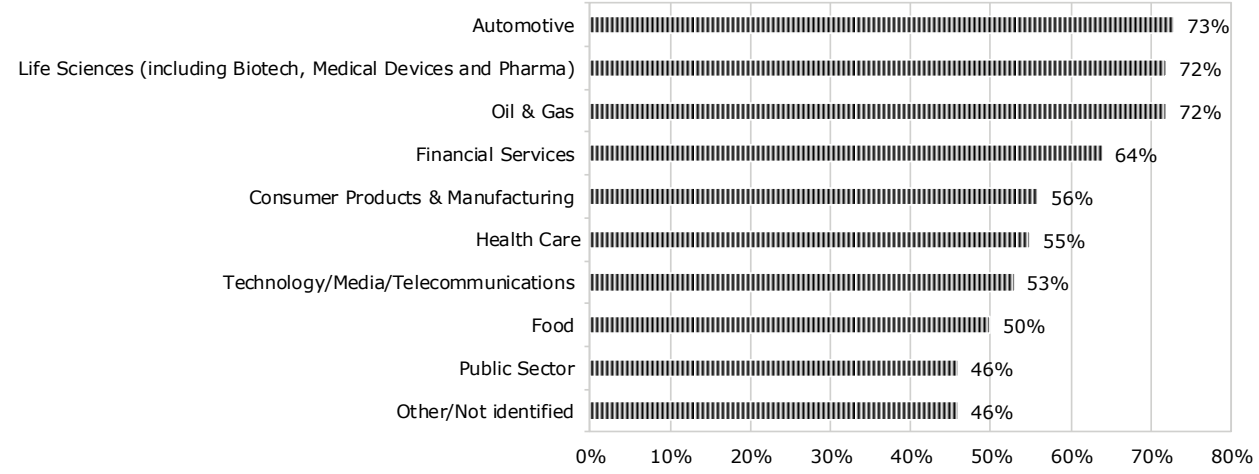

Source: Deloitte 2018 Global Blockchain Survey

Fig. 1. Answers to the question 'Blockchain technology will disrupt my organization's industry

- What is your level of agreement or disagreement with this statement regarding blockchain technology?', \% of respondents, who agree with the statement

The Public sector being a laggard for DLT deployment globally is explained by the fact that there are very few governments that mainstream and officially encourage development of DLT use cases. Examples of such countries are the United Kingdom with its public office supporting DLT start-ups financially, Estonia experimenting with DLT use cases for verification of citizens' records on government databases (E-Estonia, 2018), the United Arab Emirates working towards developing applications for government transactions being implemented predominantly through blockchain by 2021 (Smart Dubai, 2018) and Sweden with its blockchain land registry project based on blockchain. Despite the fact that The European Commission supported the signing of the European Blockchain Partnership by 27 EU Member States and established the European Blockchain Observatory to undertake research on blockchain, it is yet too early to comment on EU readiness to streamline DLT applications.

When analysing DLT use cases it is important to understand that three types of organizations are pursuing DLT innovations: established companies, start-ups and institutions. Certainly, it is more difficult for an established company or institution to fundamentally change its operating model and 
rearrange processes to match DLT functionalities that require investments, reorganizations, layoffs, new hires and completely new business philosophy. It is much easier for start-ups to streamline DLT use cases, whose whole business models are inspired by DLT.

According to Rogers' innovation adopter categories (Rogers, 1962) DLT start-ups (and probably some established companies and/ or institutions that participate in DLT consortia) can be classified as 'innovators', whose risk tolerance allows them to develop and adopt technologies that may ultimately fail and the tech and enthusiast community around DLT can be classified as 'early adopters', who make a more judicious choice of innovation adoption than innovators, but are still ready to take risk of failure. However, start-ups and early adopters are not enough to boost DLT diffusion globally, thus, established companies and/ or institutions are prerequisite to reach Rogers' 'early majority' category, who usually adopt an innovation after a varying degree of time that is significantly longer than the innovators and early adopters. 'Early majority' for DLT use cases are likely to be established companies and/ or institutions, that will integrate DLT into an already existing operational paradigm, and the final beneficiaries, who will ultimately use end products and services, that will be reshaped through DLT.

There are many examples of start-ups that reshaped traditional business models through business enabling technologies that were perceived with a degree of skepticism at the time of their occurence and are now considered success stories. Such examples include Airbnb, Uber, Carguru, Taxify, Couchsurfing, etc. that streamlined online payments and online reservations for creating new business models in transportation and hospitality industries. In this light, streamlining DLT can bring similar effects and challenge traditional business models across an array of industries. Thus, for established companies it is important to look at DLT not only from a proof of concept angle, but also from its game changing capabilities. Hesitance to pursue certain DLT use cases are associated not only with the need to fund DLT development, but also with barriers related to regulatory uncertainty, potential security threats, necessity to reorganize legacy systems, and vague return on investment.

Adoption of DLT use cases can certainly be motivated by cost savings due to operational efficiencies, since private record-keeping becomes obsolete, when all transactions are recorded in a distributed ledger, which is shared across the network. Reconciliation of transactions across private ledgers takes a lot of time and human intervention (Iansiti and Lakhani, 2017), whilst the cost of simple transactions such as invoice processing might be decreased by as much as $80 \%$ in distributed networks (IBM, 2017).

Many companies in Mexico, China, France and least in the United States are replacing parts or all of their existing systems with blockchain-based enhancements through either integrating blockchain into core operations or building new applications (Deloitte, 2018). The principles of instantaneous digital transferability of assets and the real-time confirmability of identity credentials enable new modes of contracting between parties and new forms of money (Swan et al., 2019). For example, the concept of integrated supply chain ledgers assumes that distributed ledger transactions from approved trade partners can automatically post to ledger accounts of involved parties (Swan, 2018) allowing for better financial control and risk management.

The technology sector is the most prominent for deploying DLT use cases due to vivid activity of DLT tech start-ups. For media and entertainment companies, blockchain can help track and monetize content, address piracy, and manage digital assets from creator to consumer as well as solve such challenges as simplifying tracking content usage and returning royalties to rights holders with DLT use cases for identifying, recording, and settling content interactions wherever they happen (Deloitte, 
2018). For the telecom industry, blockchain can simplify billing systems, decrease roaming fraud, create decentralized and immuTable records for accounting and audits, and enable more dynamic and flexible next-generation network services, ultimately enabling telecom operators to sell Identity as a Service (IDaaS) for customers and connected devices (Deloitte, 2018). Cloud providers are adding blockchain development tools to their platform service offerings, and chipmakers are creating specialized graphics processing units (GPUs) and application-specific integrated circuits (ASICs), start-ups and independent consortia are also working to develop blockchain solutions for identity, value exchange, and open models that anchor content rights to creators (Deloitte, 2018). These developments can disrupt the framework for provision of online services since established companies will lose its key role in content and identity management.

In Agriculture and food sector, blockchain could be used to enhance the traceability and reduce food-fraud by recording validated information concerning the origin and the state of the food - on one hand, all parties in the value chain (farmers, suppliers, processors, distributors, retailers, consumers and regulators) can access real-time information about the food product (e.g. to trace contaminated goods to its source, verify that food is produced without child labour etc.), on the other hand, users will be able to trace-back the entire product lifecycle from the farm to a retailer (ILNAS, 2018). From the food safety perspective, during contamination fast traceability of food origins can save lives. Walmart, which sells 20 per cent of all food in the U.S., conducted a traceback test on mangoes in one of its stores, that took six days, 18 hours, and 26 minutes to trace mangoes back to its original farm, whilst by using blockchain, Walmart can provide all the information the consumer wants in 2.2 seconds (Charlebois, 2018).

The Blockchain In Transport Alliance (BiTA), has quickly grown into the largest commercial blockchain alliance in the world that leads the effort to develop and embrace a common framework and standards from which transportation/ logistics/ supply chain/ freight marketplace participants can build revolutionary blockchain and DLT applications (BiTA, 2018). The logistics giant Fedex is actively testing ways to use blockchain to track high-cost, mission-critical cargo (Mathis, 2018).

In its automotive industry research Deloitte has developed three use case groups to cover the breadth of the automotive market and blockchain application based on the analysis of over 40 use cases: Verification and process improvements, Vehicle management and incentives and Finance, payments and insurance (Deloitte, 2018).

The Construction Blockchain Consortium (CBC) supports knowledge transfer, arranges commercial and academic presentation, assesses and tests commercial services and technology, conducts research, and works on relevant case studies and proof of concept in Building information modelling, Internet of things and Machine Learning (CBC, 2018). CBC also works towards solutions to reduce incidences of parties suing one another for shoddy work or delays in project completion (Rothrie, 2018). Probuild, one of Australia's largest building firms, has partnered with US blockchain construction innovator Brickschain for managing its global supply chain (Coincentral, 2018).

Deloitte has identified six areas for improving the leasing and purchase and sale process of real estate through the use of blockchain: improve property search process, expedite pre-lease due diligence, ease leasing and subsequent property and cash flow management, enable smarter decision-making, transparent and relatively cheaper property title management, enable more efficient processing of financing and payments (Deloitte, 2017). The actual use cases include such start-ups as Propy, which allows investors to purchase property through blockchain in a variety of locations and aids cross-border property transactions, Streetwire, a clearinghouse for real estate 
data and transactions, which streamlines processes around closing, lending and valuing property while returning value and control to data producers and many others (Dunn, 2018).

According to the Deloitte 2018 Global Blockchain survey most of the CEE respondents have not yet started to invest into Blockchain technology, only one quarter of the respondents claimed to have made investments so far, the existing investments have been manifested mostly in the Financial services and Technology/Media/Telecommunication sectors with some investments being made in Consumer Products \& Manufacturing and Energy \& Resources sectors and the existing use cases are mostly related to digital recording and payment solutions, but there are prototypes under construction for digital identity and also for supply chain traceability (Deloitte, 2018). There is a logical distribution of the use cases in line with the main profile of the sectors: supply chain solutions are mostly popular in the Consumer products \& Manufacturing sector.

\section{Analysis of sectors subject to disruption by DLT within the economy of Latvia}

In 2018 Latvia together with other European countries signed the declaration of 'Cooperation on a European Public Blockchain Partnership', which aims to develop a trusted, secure and resilient European Blockchain Services Infrastructure (EBSI) meeting the highest standards in terms of privacy, cybersecurity, interoperability and energy efficiency, as well as fully compliant with EU law (EC, 2018). This signature signals that the government of Latvia is aware of DLT and will further be involved in experience exchange and DLT use case development activities on EU level. These developments reflect that Latvia has reached the $3^{\text {rd }}$ stage of innovation decision-making process titled 'Decision' as suggested by Rogers' innovation diffusion theory (Rogers, 1962), meaning that the government of Latvia currently weighs advantages and disadvantages of using DLT.

Due to non-existence of use cases beyond crypto-currencies and ICOs in Latvia and the indecisiveness of the government of Latvia about willingness or unwillingness to support distributed ledger applications, it is impossible to analyse disruption potential based on practical examples, thus, it is necessary to assess potential for emergence of such cases in Latvia in short, medium or long term. The time frame cannot yet be precisely quantified and should be interpreted relatively to the global time frime, since DLT use cases (apart from crypto-currencies and ICOs) have not yet reached the 'early majority adopter category' globally or regionally as per Rogers' classification of individuals within a social system on the basis of innovativeness (Rogers, 1962).

In order to identify sectors with significant contribution, intermediate consumption and gross value added accounts are used as indicators for assessing activity related to exchange of goods and services within particular sector (as per Nace 2 classification). Intermediate consumption is an indicator for B2B activity, whilst value added is an indicator of B2C activity, both subject to disruption by DLT.

Sectors with the most significant contribution to the economy of Latvia measured by output (trespassing a $5 \%$ weight in the total output) are Manufacturing, Transportation and storage, Wholesale and retail trade, repair of motor vehicles and motorcycles, Construction, Real estate activities, Agriculture, Forestry and Fishing, Public administration and defence, compulsory social security, and Information and communication.

Manufacturing is the most significant sector within the economy of Latvia that constituted $17.8 \%$ of total output, $22.9 \%$ of total intermediate consumption and $14.6 \%$ of total gross value added in 2017. The research has identified the existence of DLT use cases for supply chain traceability in the manufacturing sector within the Central and Eastern Europe. Since those applications are already being developed within the CEE region, it is only a matter of time when those applications will reach 
Latvia, thus, distributed ledger solutions for supply chain traceability are potential disruptors for the Latvian manufacturing sector in the medium term.

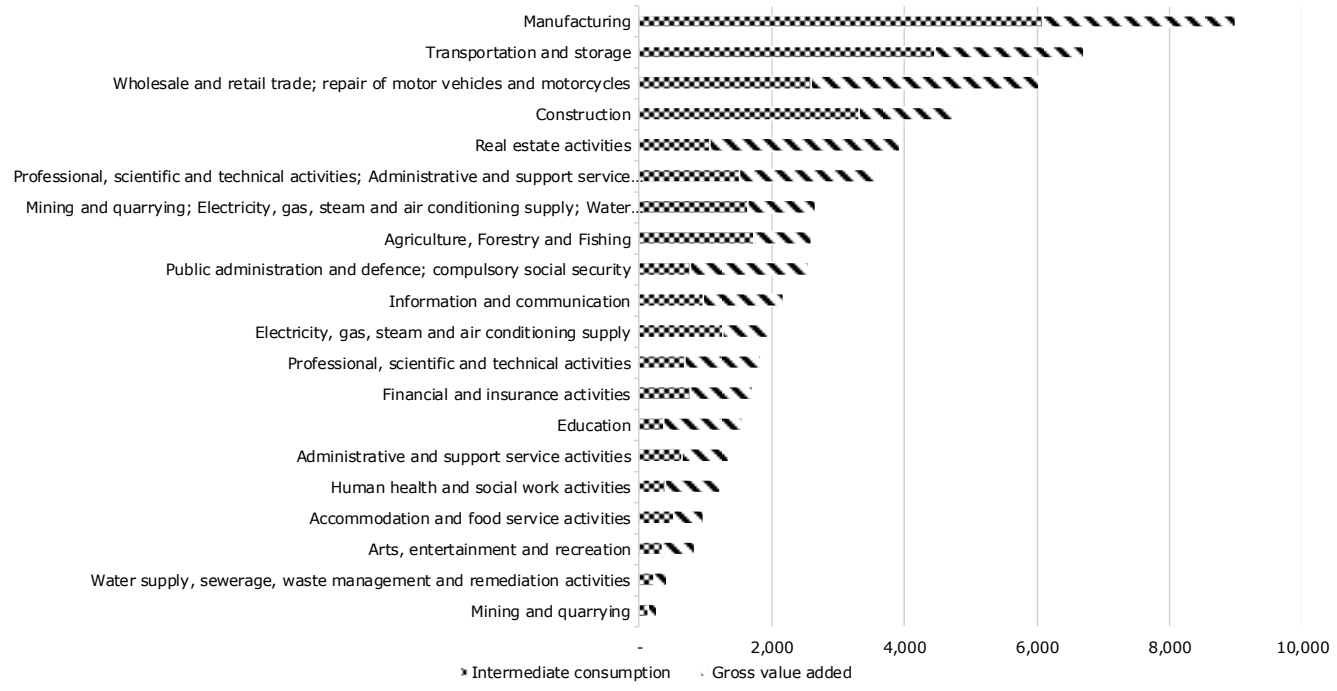

Source: author's calculations based on Central Statistical Bureau of Latvia

Fig. 2. Intermediate consumption and gross value added per sector in Latvia at current prices in 2017, MEUR

Transportation and storage sector constituted $13.4 \%$ of total output, $16.7 \%$ of total intermediate consumption and $12.2 \%$ of total gross value added in 2017. The Blockchain In Transport Alliance is actively working on developing industry standards deploying DLT technology, thus, potential DLT solutions related to administrative improvements, trade finance, dispute resolution, load planning and distribution and freight tracking are potential disruptors to Transportation and storage sector in Latvia in the long term when the whole global Transportation sector will adopt DLT standards. Global DLT adoption is prerequisuite since Transportation involves cross-border transactions being implemented by multiple stakeholders, which makes it virtually impossible for single or couple major players to reinvent such complicated and mutually reliant business processes.

Wholesale and retail trade, repair of motor vehicles and motorcycles constituted $12.0 \%$ of total output, $12.4 \%$ of total intermediate consumption and $12.1 \%$ of total gross value added in 2017 . The research has identified the existence of distributed ledger use cases for supply chain traceability in the Consumer products sector within the Central and Eastern Europe. Since those applications are already being developed within the CEE region, it is only a matter of time when they will reach Latvia, thus, distributed ledger solutions for supply chain traceability are potential disruptors for the Latvian Wholesale and retail trade sector in the medium term. Deloitte research on the automotive industry records development of at least 40 use cases globally, thus, solutions for supply chain traceability and vehicle information improvements are potential disruptors for Repair of motor vehicles and motorcycles sector in the long term, when at least one leading automotive producer will implement DLT within its supply chain.

Construction sector constituted $9.4 \%$ of total output, $9.7 \%$ of total intermediate consumption and $9.5 \%$ of total gross value added in 2017. The Construction Blockchain Consortium is actively working on developing DLT use cases as well some companies are fostering their own use cases, thus, potential DLT solutions related to supply chain traceability, dispute resolution and operational improvements are potential disruptors to Construction sector in Latvia in the long term. However it is also theoretically possible for local construction companies to develop their standalone DLT use cases through applications related to supply chain traceability since Construction sector is not as 
global as Transportation, thus, it is possible to reorganize business processes within a supply chain of one particular company (subject to technological savviness and business rationale).

Real estate activities constituted $7.8 \%$ of total output, $6.5 \%$ of total intermediate consumption and $7.6 \%$ of total gross value added in 2017. The research has identified areas in the real estate sector that can be improved through DLT as well as several use cases outside the CEE region have been identified, thus, potential DLT solutions related to improving the leasing and purchase and sale processes are potential disruptors to Real estate activities sector in Latvia in the long term. However, it is also theoretically possible for local real estate companies to develop local DLT use cases through applications related to purchase/ sale and leasing transactions, since real estate activities can be managed locally (subject to technological savviness and business rationale).

Agriculture, Forestry and Fishing constituted $5.2 \%$ of total output, $4.8 \%$ of total intermediate consumption and $6.1 \%$ of total gross value added in 2017 . The research has identified areas in the Agriculture sector that can be improved through DLT as well as several use cases outside the CEE region have been identified, thus, potential DLT solutions related to improving food safety and supply chain traceability are potential disruptors to Agriculture, Forestry and Fishing sector in Latvia in the long term. However, it is also theoretically possible for local farmers to develop local DLT use cases through applications related to storing data about production life cycle of agricultural products (subject to technological savviness and business rationale).

Public administration and defence; compulsory social security constituted $5.1 \%$ of total output, $4.0 \%$ of total intermediate consumption and $5.0 \%$ of total gross value added in 2017 . The research has identified areas in the Public sector that can be improved through DLT, several country's efforts to streamline DLT adoption, including neighbouring Estonia as well as Latvia's participation in European Blockchain Partnership, however, potential DLT solutions related to citizen's identity management, management of land/assets, inter- or intragovernmental transactions and sharing health records are potential disruptors to Public administration sector in Latvia in the long term taking into account higher public sector rigidness to innovation and Latvia's position as an e-government laggard among Baltic states (Kostrikova, Rivza, 2017).

Information and communications (ICT) sector constituted $4.3 \%$ of total output, $3.7 \%$ of total intermediate consumption and $5.0 \%$ of total gross value added in 2017 . The research has identified areas in the IT and Telecom sectors that can be improved through DLT as well as several use cases in Technology/Media/Telecommunication sectors have been identified in the CEE region. Also, there are already some start-ups in Latvia that develop DLT solutions in the ICT sector such as Digipulse digital asset inheritance platform, Aeternum - platform, through which every individual can invest in intellectual property rights of scientists around the world, Notakey - solution for identity verifications and Know-Your-Customer checks and others. Identified use cases signal that ICT sector in Latvia has already reached the $4^{\text {th }}$ stage of innovation decision making process titled 'Implementation' (Rogers, 1962), when DLT is being employed in practice. Thus, potential DLT solutions related to improving identity, digital content and copyright management are potential disruptors to ICT sector in Latvia in the short term.

\section{Conclusions, proposals, recommendations}

1) Global leaders acknowledge the disruptive potential of DLT and its capabilities to fundamentally alter existing business models. The level of awareness and readiness is lower in the CEE region comparing to global trends. Many market leaders participate in various blockchain inspired consortia that aim towards development of DLT based industry standards and DLT use cases. 
However, in practice there are very few functioning DLT use cases beyond crypto-currencies and ICOs. Companies and governments globally invest in developing such DLT use cases with the majority of use cases being deployed in the developed countries. None of such use case has yet reached the early majority adoption stage.

2) Identified economic sectors with the significant value added within the economy of Latvia are all subject to disruption from DLT in the short, medium or long term. The short-term disruption potential from DLT has been identified in the Information and communications sector. The medium-term disruption potential has been identified in Manufacturing, Wholesale and retail trade and Public administration sectors. The long-term disruption potential has been identified in Transportation, Repair of motor vehicles and motorcycles sector, Construction, Real estate activities and Agriculture, Forestry and Fishing sectors.

3) Further research will focus on quantifying potential economic impact from deploying DLT use cases within identified economic sectors of substantial weight within the economy of Latvia as well as assessing indirect effects from sectors with lesser weights within the economy of Latvia.

\section{Acknowledgements}

The preparation of the paper was supported by the National Research Programme 5.2. Project „INTERFRAME-LV”.

\section{Bibliography}

1. Anand, S. (2018). A Pioneer In Real Estate Blockchain Emerges In Europe. The Wall Street Journal. Retrieved: https://www.wsj.com/articles/a-pioneer-in-real-estate-blockchain-emerges-in-europe1520337601 Access: 15.02.2019.

2. Blockchain in Transport Alliance (2018). Retrieved: https://www.bita.studio/ Access: 15.02.2019.

3. Central Statistical Bureau of Latvia (2018). Retrieved: https://www.csb.gov.Iv Access: 10.02.2019.

4. Charlebois, S. (2018). How Blockchain Technology Could Transform The Food Industry. Retrieved: https:// theconversation.com/how-blockchain-technology-could-transform-the-food-industry-89348 Access: 12.02.2019.

5. Construction Blockchain Consortium (2018). Retrieved: https://www.constructionblockchain.org/ Access: 15.02.2019.

6. Deloitte (2018). Breaking Blockchain Open. Deloitte's 2018 Global Blockchain Survey. Retrieved: https://www2.deloitte.com/content/dam/Deloitte/cz/Documents/financial-services/cz-2018-deloitte-globalblockchain-survey.pdf Access: 02.02.2019.

7. Deloitte (2018). Breaking Blockchain Open. Central and Eastern European Perspective. Retrieved: https://www2.deloitte.com/content/dam/Deloitte/ce/Documents/about-deloitte/ce-blockchain-survey-2018central-europe-perspective.pdf Access: 10.02.2019.

8. Deloitte (2018). Accelerating Technology Disruption In The Automotive Market. Blockchain in the Automotive Industry. Retrieved:

https://www2.deloitte.com/content/dam/Deloitte/cn/Documents/consumer-business/deloitte-cn-consumerblockchain-in-the-automotive-industry-en-180809.pdf Access: 07.02.2019.

9. Deloitte (2017). Blockchain in Commercial Real Estate: The Future Is Here. How Blockchain-based Smart Contracts Could Revolutionize Commercial Real Estate. Retrieved: https://www2.deloitte.com/content/dam/Deloitte/us/ Documents/financial-services/us-dcfs-blockchain-incre-the-future-is-here.pdf Access: 11.02.2019.

10.Dunn, J. (2018). 4 Blockchain Real Estate Startups Shaking Up Property Investment. Retrieved: https://espeoblockchain.com/blog/blockchain-real-estate-startups/ Access: 15.02.2019.

11. E-estonia (2018) Retrieved: https://e-estonia.com/solutions/e-identity/id-card/ Access: 15.02.2019.

12. European Commission (2018). European Countries Join Blockchain Partnership. Retrieved: https://ec.europa.eu/ digital-single-market/en/news/european-countries-join-blockchain-partnership Access: 15.02.2019.

13. Iansiti, M. and Lakhani, K. R. (2017). The Truth About Blockchain. Harvard Business Review 95(1). pp. 118 $-127$

14. IBM (2017). Boosting Financial Intelligence With Cognitive Computing. Retrieved: https://economia.icaew.com/ features/ july-2017/boosting-financial-intelligence-with-cognitive-computing Access: 12.02 .2019

15. ILNAS - Institut Luxembourgeois de la Normalisation, de l'Accréditation, de la Sécurité et qualité, Agence pour la Normalisation et l'Economie de la Connaissance des produits et services (2018). White Paper. Blockchain And Distributed Ledgers Technology, Economic Impact And Technical Standardization. Retrieved: 
https://portail-qualite.public.lu/dam-assets/publications/normalisation/2018/white-paper-blockchain-june2018.pdf Access: 05.02.2019.

16. Kostrikova, N., Rivza, B. (2017). E-government And E-participation In Baltic States: Comparison of Estonia, Latvia, Lithuania in Proceedings of the 2017 International Conference "ECONOMIC SCIENCE FOR RURAL DEVELOPMENT" No 45 Jelgava: LLU ESAF. pp. 118-126.

17. Mathis, J. (2018). FedEx Is Testing Blockchain Tech For Critical Cargo Shipments. Retrieved: https://www.ccn.com/tracking-key-shipments-fedex-is-testing-with-blockchain Access: 12.02.2019.

18. Rogers, E. M. (1962). Diffusion Of Innovations. New York: Free Press of Glencoe. pp 172-175, p. 249.

19. Rothrie S. (2018). How Blockchain And Construction Will Build A New World. Retrieved: https://coincentral.com/blockchain-and-construction/ Access: 08.02.2019.

20.Smart Dubai (2018) Retrieved: http://smartdubai.ae Access: 07.02.2019.

21.Swan, M. (2018). Blockchain Economics: Ripple For ERP Integrated Blockchain Supply Chain Ledgers. Integrated Supply Chain Ledgers To Free \$3.9 Trillion In Capital? The European Financial Review. pp. 24-26.

22.Swan, M. et al. (2019). Blockchain Economics: Implications Of Distributed Ledgers - Markets, Communications Networks And Algorithmic Reality. London: World Scientific Publishing Europe. p. 6 